\title{
Interplay between Polar Distortions and Superconductivity in $\mathrm{SrTiO}_{3}$
}

\author{
Salva Salmani-Rezaie, Hanbyeol Jeong, Kaveh Ahadi and Susanne Stemmer
}

University of California-Santa Barbara, United States

$\mathrm{SrTiO}_{3}$ is the first oxide superconductor discovered, but the nature of superconductivity is still heavily debated in this material. $\mathrm{SrTiO}_{3}$ shows a superconducting state in extremely dilute regimes, in which Debye temperature is significantly larger than Fermi temperature and superconductivity is at odds with Bardeen-Cooper-Schrieffer description. Superconductivity frequently emerges near an electronic order in unconventional superconductors. Superconductivity in $\mathrm{SrTiO}_{3}$ occurs near a polar instability and recent observations suggest a direct link between the enhancement of and ferroelectric Curie temperature [1], [2]. Tuned $\mathrm{SrTiO}_{3}$ shows polar superconductivity in which spin-orbit coupling can result in an unconventional superconducting state[3]-[5]. Sensitivity to disorder is a probe to reveal the nature of superconductivity in quantum materials. Conventional superconductors are protected against nonmagnetic disorders. Atomic resolution high-angle annual dark-field imaging in scanning transmission electron microscopy (HAADF-STEM) is a powerful technique that is capable of detecting subtle structural disorders.

In this study, we use HAADF-STEM to measure Ti column displacement vectors in the Sm-doped compressively strained $\mathrm{SrTiO}_{3}$ thin films. These films undergo successive ferroelectric and superconducting transitions. $\mathrm{SrTiO}_{3}$ thin films were grown using hybrid molecular beam epitaxy (MBE) on (001) LSAT single crystals. Films were doped with Sm with carrier densities of $n_{3 D}$ of $6 \times 10^{19} \mathrm{~cm}^{-3}$, $1.4 \times 10^{20} \mathrm{~cm}^{-3}$, and $2.8 \times 10^{20} \mathrm{~cm}^{-3}$. HAADF-STEM images obtained at room temperature and polarization vector assigned to each $\mathrm{Ti}$ column. The polarization vector is defined as the difference between the center of mass of the four neighboring Sr columns and the Ti column position obtained by 2D Gaussian fitting.

Figure 1(a) and (b) show the direction of polarization vector for two Sm-doped samples with $n 3 D$ of $6 \times 10^{19} \mathrm{~cm}^{-3}$, and $2.8 \times 10^{20} \mathrm{~cm}^{-3}$, respectively. The color wheel shows the polarization direction in 30 degree intervals. The large clusters of red vectors indicate the formation of [001] nano-domains on the low doped sample which undergoes ferroelectric transition at $92 \mathrm{~K}$ and becomes superconducting at $~ 600$ $\mathrm{mK}$. The displacements of Ti-columns become completely random with increasing the carrier density to $2.8 \times 10^{20} \mathrm{~cm}^{-3}$. The polar phase does not set in for this sample and becomes superconducting at $\sim 200 \mathrm{mK}$. Sm dopant atoms introduce lattice distortions, destabilizing the cooperative Ti displacements and, as a result, suppress the ferroelectric transition and superconductivity. Figure 2 shows the distribution of the magnitude of the displacements measured for doped and undoped strained $\mathrm{SrTiO}_{3}$ obtained from $7000 \mathrm{Ti}$ columns.

The undoped strained $\mathrm{SrTiO}_{3}$ films have the largest polar distortions associated with large polar domains and high Curie temperature. Increasing the dopant concentration suppresses the average magnitude of the polar distortions until the strain field of dopants overlap and distortion increases. Here, we observe a systematic suppression of the correlated polar distortions and ferroelectricity with the introduction of the Sm dopants. We also observe an inverse relationship between the critical temperature of superconductivity and the magnitude of polar distortions. The peak of the superconductivity dome corresponds to the smallest polar distortions, highlighting the sensitivity of superconductivity to nonmagnetic disorder in $\mathrm{SrTiO}_{3}$. The interaction of magnetic disorders and superconductivity in $\mathrm{SrTiO}_{3}$ provides insight into the nature of the superconducting state [6]. 


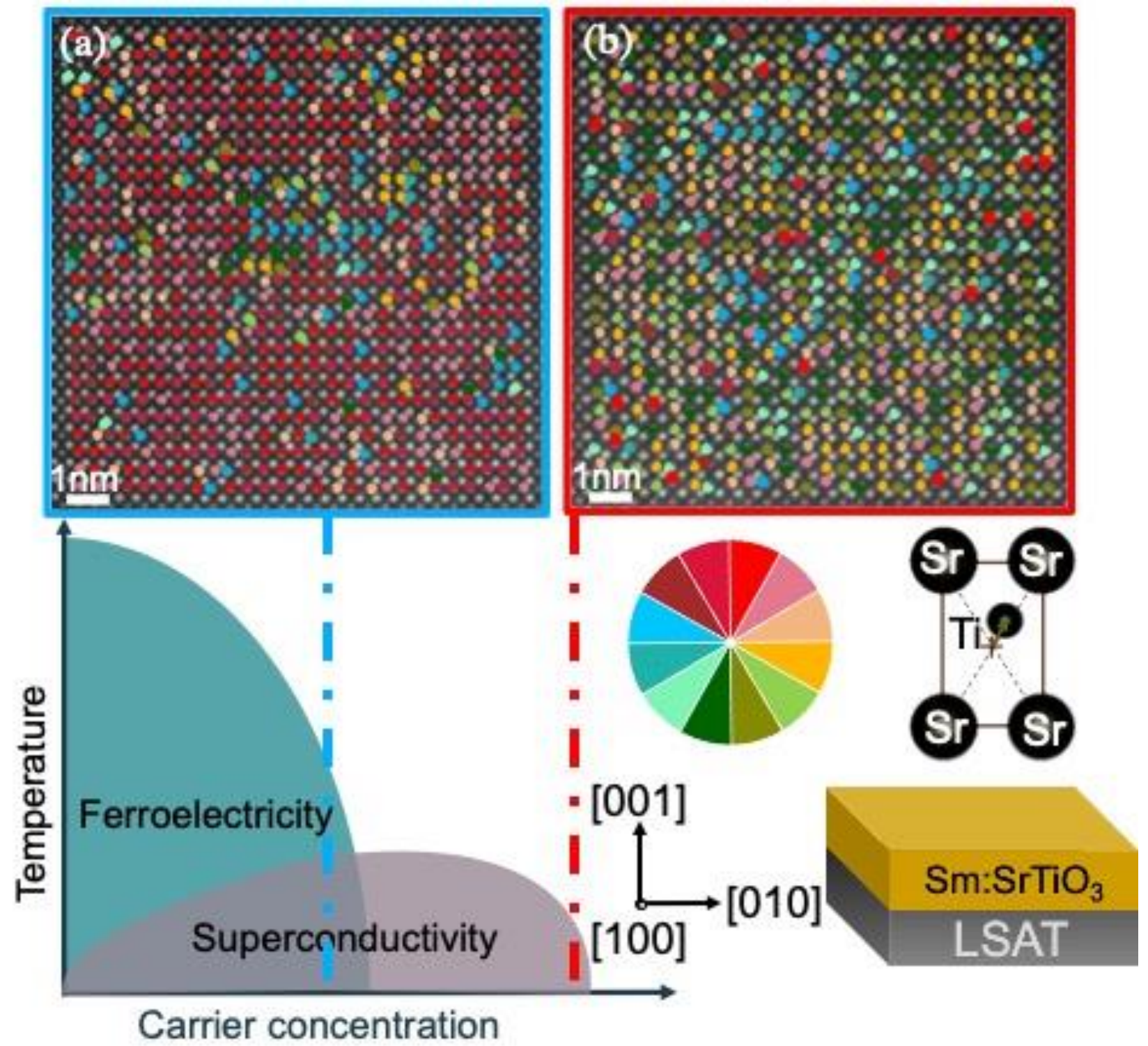

Figure 1. Figure 1- HAADF-STEM images of polarization maps of epitaxially strained Sm doped SrTiO3 films with n3D of (a) $6 \cdot 1019 \mathrm{~cm}-3$ and (b) $2.8 \times 1020 \mathrm{~cm}-3$ Increasing the doping, polar domains shrink and superconductivity and ferroelectricity get suppressed. 

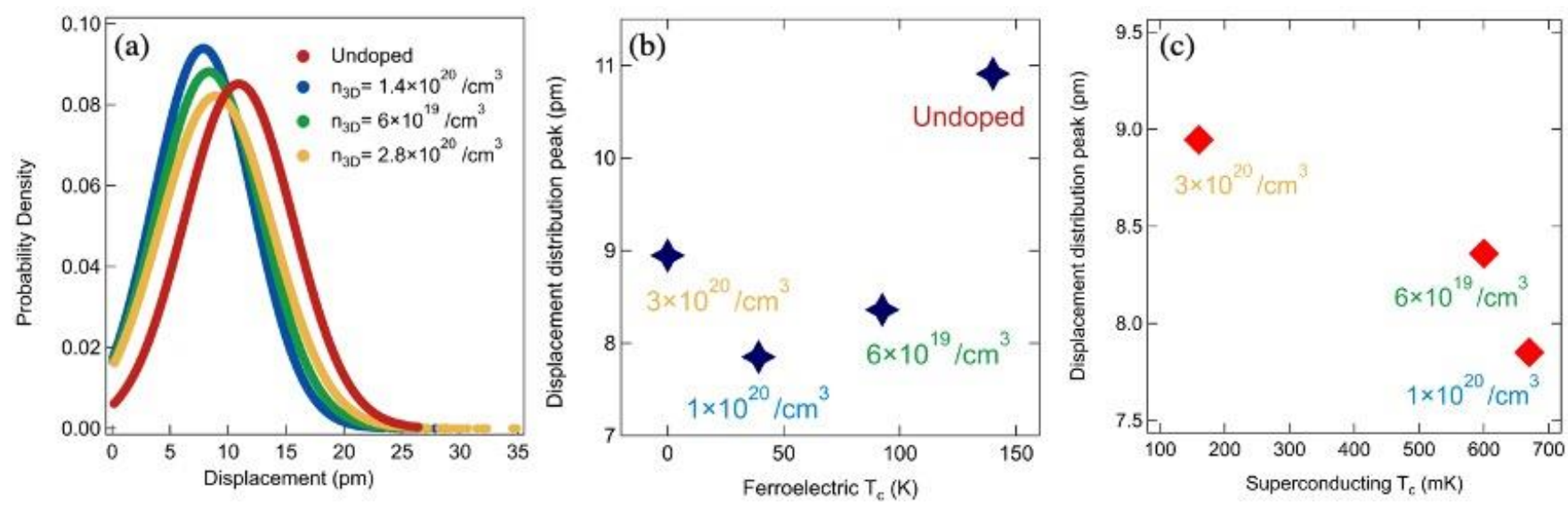

Figure 2. Figure 2 (a)Distribution of the magnitude of the displacements at room temperature (b) change in Curie temperature with the average magnitude of the displacements (c) Superconducting critical temperature as a function of the average magnitude of the displacement

\section{References}

[1] K. Ahadi, L. Galletti, Y. Li, S. Salmani-Rezaie, W. Wu, and S. Stemmer, "Enhancing superconductivity in SrTiO3 films with strain," Sci. Adv., vol. 5, no. 4, 2019, doi: 10.1126/sciadv.aaw0120.

[2] R. Russell, N. Ratcliff, K. Ahadi, L. Dong, S. Stemmer, and J. W. Harter, "Ferroelectric enhancement of superconductivity in compressively strained SrTiO 3 films," Phys. Rev. Mater., vol. 3, no. 9, p. 091401, Sep. 2019, doi: 10.1103/PhysRevMaterials.3.091401.

[3] S. Salmani-Rezaie, K. Ahadi, and S. Stemmer, "Polar Nanodomains in a Ferroelectric Superconductor," Nano Lett., vol. 20, no. 9, pp. 6542-6547, Sep. 2020, doi: 10.1021/acs.nanolett.0c02285.

[4] S. Salmani-Rezaie, K. Ahadi, W. M. Strickland, and S. Stemmer, "Order-Disorder Ferroelectric Transition of Strained SrTiO3," Phys. Rev. Lett., vol. 125, no. 8, p. 087601, Aug. 2020, doi: 10.1103/PhysRevLett.125.087601.

[5] T. Schumann et al., "Possible signatures of mixed-parity superconductivity in doped polar SrTi O 3 films," Phys. Rev. B, vol. 101, no. 10, p. 100503, Mar. 2020, doi: 10.1103/PhysRevB.101.100503. [6] The authors acknowledge support by the U.S. Department of Energy (Grant No. DEFG02- 2ER45994). 